\title{
Analyzing the EEG Signals in Order to Estimate the Depth of Anesthesia Using Wavelet and Fuzzy Neural Networks
}

\author{
Mansour Esmaeilpour ${ }^{1}$, Ali Reis Ali Mohammadi² \\ ${ }^{I}$ Computer Engineering Department, Hamedan Branch, Islamic Azad University, Hamedan, Iran
${ }^{2}$ Computer system architecture Department, Arak Branch, Islamic Azad University, Arak, Iran
}

Abstract - Estimating depth of Anesthesia in patients with the objective to administer the right dosage of drug has always attracted the attention of specialists. To study Anesthesia, researchers analyze brain waves since this is the place which is directly affected by the drug. This study aimed to estimate the depth of Anesthesia using electroencephalogram (EGG) signals, wavelet transform, and adaptive Neuro Fuzzy inference system (ANFIS). ANFIS can estimate the depth of Anesthesia with high accuracy. A set of EEG signals regarding consciousness, moderate Anesthesia, deep Anesthesia, and iso-electric point were collected from the American Society of Anesthesiologists (ASA) and PhysioNet. First, the extracted features were combined using wavelet and spectral analysis after which the target features were selected. Later, the features were classified into four categories. The results obtained revealed that the accuracy of the proposed method was $98.45 \%$. Since the visual analysis of EEG signals is difficult, the proposed method can significantly help anesthesiologists estimate the depth of Anesthesia. Further, the results showed that ANFIS could significantly increase the accuracy of Anesthesia depth estimation. Finally, the system was deemed to be advantageous since it was also capable of updating in real-time situations as well.

Keywords - Anesthesia, Electroencephalogram, Wavelet, Classification, Adaptive Neuro Fuzzy Inference System.

\section{INTRODUCTION}

A NESTHESIA results from administration of anesthetic drugs, through injection, and is characterized by sleepiness and loss of feeling of pain. Today, depth of Anesthesia is expressed as a consistent decrease in the performance of Central Nervous System (CNS) as well as decreased response to stimuli $[1,2]$. Injecting an appropriate level of anesthetic drugs has always been a great concern to anesthesiologists. They seek ways to ensure patients' blood circulation and, at the same time, increase the depth of Anesthesiato ensures patients' recovery in a shorter period of time. Since the most important causes of Anesthesia lie in the brain, researchers mostly focus on analyzing the Electroencephalogram(EEG) [2,3].Recently, methods basing on EEG signals - these signals express the electrical activity of the brain and are dynamic, random, non-stop, and non-linear [4,5] - have increased the accuracy of Anesthesia depth estimation. These methods are particularly efficient for separating nonstop signals with their impedance and Anesthesia. An expert needs to visually analyze a large amount of EEG signals to extract the required information. The computer analysis of EEG signals aims at making such data extraction fast, effortless, and automatic.

Mathematical models, i.e. artificial neural networks and fuzzy systems, have an array of applications in clinical medicine - extraction of features and diagnosis of illness being only few examples. Fuzzy systems are modeling methods with wide applications in various scientific fields.
They are used widely and quite efficiently in investigations targeting different biological and non-biological phenomena [6,7].

Adaptive Neuro Fuzzy Inference System (ANFIS) exploits neural network and fuzzy logic algorithms to formulate a non-linear mapping between the input and the output space. Having the linguistic strength of a fuzzy system and the numerical strength of a neural network, ANFIS has proven to be particularly efficient in modeling complex processes $[6,7,8]$.Accordingly, the present study aimed at proposing an intelligent model to estimate depth of Anesthesia using features extracted from EEG signals. Sample features obtained from wavelet coefficients as well as the spectral analysis of EEG signals were used as parameters in this study.

\section{Material and Methods}

To undertake the present study, use was made of EEG signals obtained from American Society of Anesthesiologists(ASA) - and the Sleep-EDF database of PhysioNet. The signals used included four categories namely consciousness, moderate Anesthesia, deep Anesthesia, and coma. The signals collected were in BDF, EDF, ASCII, and TEXT formats. To extract features, MATLAB R2009a, EDFBrowser, and EEGLAB applications were drawn on. Figures $1 \mathrm{a}$ to $1 \mathrm{~b}$ illustrate examples of EEG signals belonging to each category respectively.

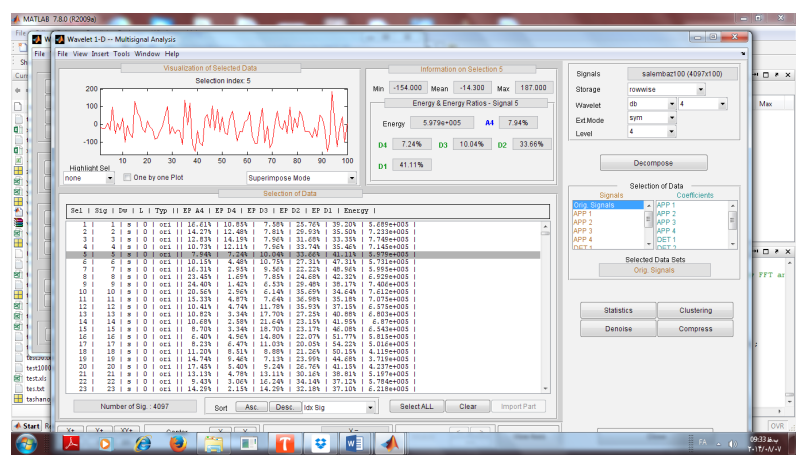

Fig. 1 a. A sample of the EEG signals in consciousness mode.

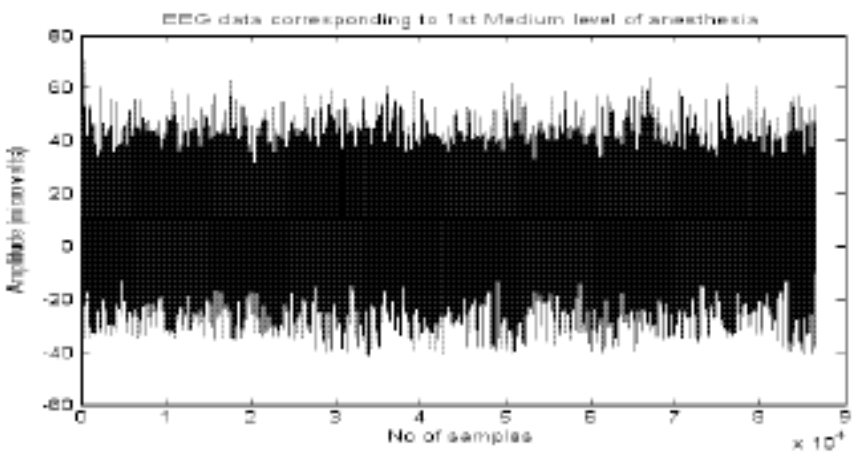

Fig. 1b. A sample of the EEG signal in the middle of Anesthesia. 
Wavelet analysis is one of the most useful methods in analyzing bio-signals, such as EEG ones [9]. For this reason Discrete Wavelet Transform (DWT) was used to extract statistical features. Wavelet transform is particularly used in extracting non-stop signals with various frequency characteristics [10]. In fact, it decomposes signals into a set of basic functions. These continuous basic functions are calculated from the application of delays, contractions, and transmissions on a unique function called wavelet model [11]. Using a Daubechies 4 (D4) wavelet transform, the collected signals are decomposed into 4 subbands. The frequency range was taken to be within 0 to $60 \mathrm{~Hz}$ and frequencies higher than $60 \mathrm{~Hz}$ were dismissed as noises. As shown in Figure 1c, the original signal is first passed through a High Pass Filter (HPF) and a Low Pass Filter (LPF).

Having removed other samples, the signals are decomposed into two simpler signal types. Each stage comprises two digital filters and two down-sampler filters. The output of the first HPF and LPF results in D1 component and A1 approximation. Then, the first calculated approximation, A1, is decomposed further which produces component D2 and approximation A2. In all, this procedure is repeated four times in this study. As shown in Figure 1d, D1, D2, D3, D4, and A4 are gamma sub-band, beta sub-band, alpha sub-band, theta sub-band, and delta sub-band, respectively.

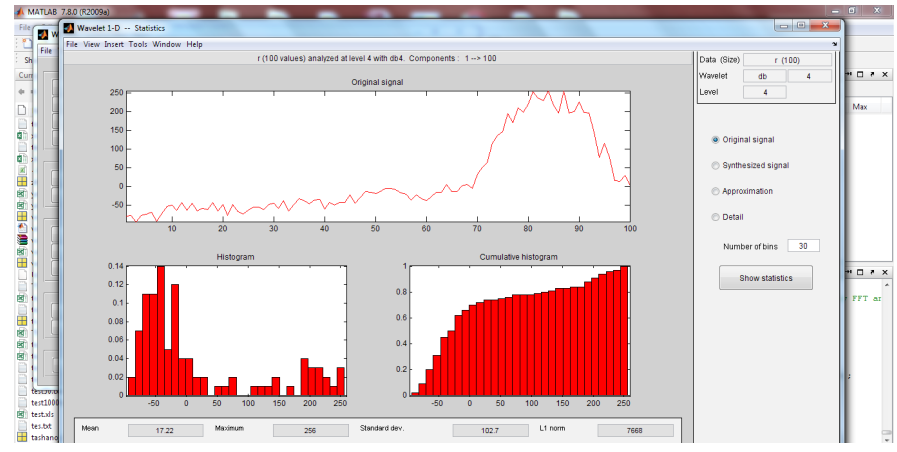

Fig. 1c. A sample of the EEG signal in a state of deep anesthesia.

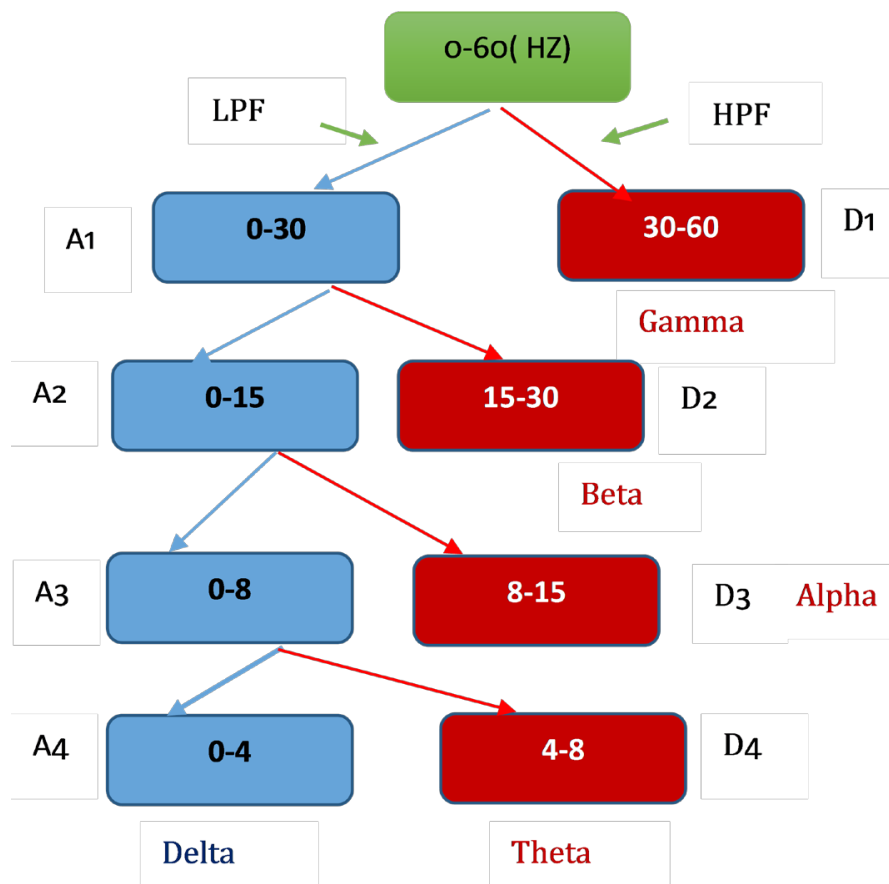

Fig. 1d. EEG signal is decomposed into sub-bands.
Since sub-bands contain more accurate information compared to the mother signal, decomposition was carried out using wavelet. Having completed this stage, the desired and optimized information and features were extracted from the sub-bands. Figures $1 \mathrm{e}$ and $1 \mathrm{f}$ show examples of decomposed signals in various levels, including consciousness and Anesthesia.

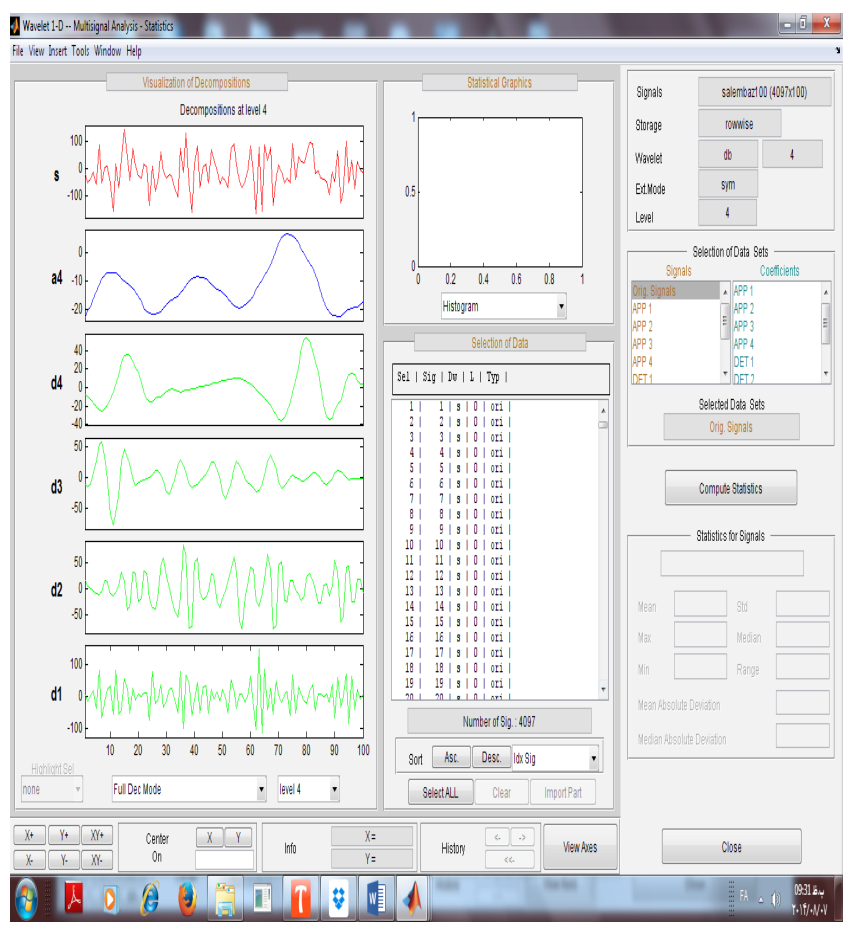

Fig. 1e. EEG signal levels in db 4to alert level 4.

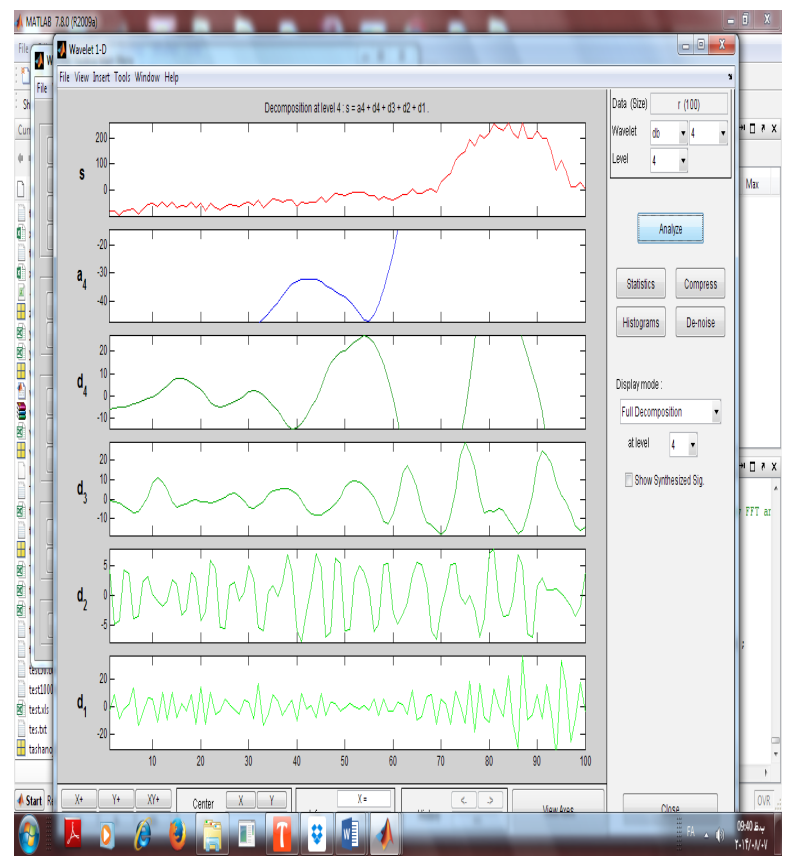

Fig. 1f. EEG signal levels in Anesthesia state to the db 4with level 4.

The target statistical features in this study included the maximum and minimum of wavelet coefficients as well as the mean and the standard deviation of each set of signals. All these features were extracted from each sub-band. Moreover, all the spectral features intended were also extracted. The calculated features included alpha and beta ratios (Eq. $2.1 \& 2.2$ ) as well as theta ratio (Eq. 2.3) denoting the depth of 
Anesthesia, consciousness, and a state between alpha and beta and between moderate Anesthesia and other states, respectively[12].

$$
\begin{aligned}
& \text { Alpha }_{\text {ratio }}=\log \frac{E(30-42.5 \mathrm{~Hz})}{E(6-12 \mathrm{~Hz})} \\
& \text { Beta }_{\text {ratio }}=\log \frac{E(30-42.5 \mathrm{~Hz})}{E(11-21 \mathrm{~Hz})} \\
& \text { Theta }_{\text {ratio }}=\log \frac{E(6-12 \mathrm{~Hz})}{E(11-21 \mathrm{~Hz})}
\end{aligned}
$$

Further, four features were extracted from the set of statistical and spectral features as indicated in Table 1.

TABLE I. The Extracted Features

\begin{tabular}{|c|c|c|}
\hline $\begin{array}{c}\text { Spectral characteristics } \\
\text { are extracted }\end{array}$ & $\begin{array}{c}\text { Statistical features } \\
\text { extracted }\end{array}$ & $\begin{array}{c}\text { Features used on this } \\
\text { model }\end{array}$ \\
\hline Alpha ratio & (MAX) & Alpha ratio \\
\hline Beta ratio & (MIN) & Beta ratio \\
\hline Theta ratio & (STD) & Theta ratio \\
\hline Gamma ratio & Mean & STD \\
\hline
\end{tabular}

These features were used as input for the designed Fuzzy Inference System (FIS)[13]. In the proposed FIS, the first input, as shown in Figure 1g, comprises factors that draw a clear cut distinction between Anesthesia and the other states. Similarly, the second input, Figure 1h, represents consciousness and the other states. In the same vein, the third input, as illustrated in Figure 1i, represents moderate Anesthesia, Anesthesia, and consciousness. Finally, Figure $1 \mathrm{j}$ distinguishes coma from other states.

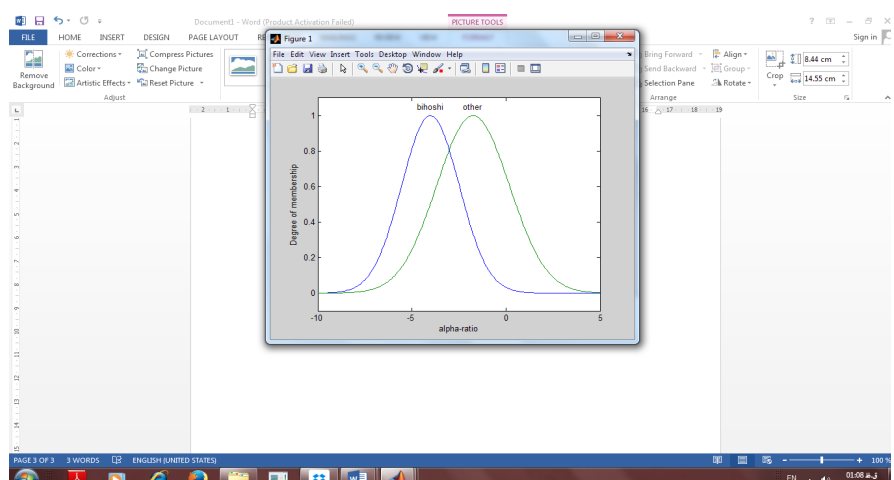

Fig. 1g. The first input in FIS.

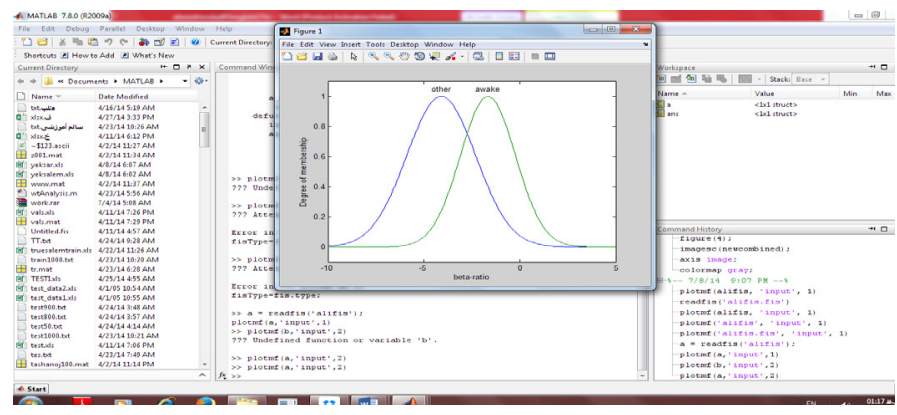

Fig. 1h. The second input in FIS.

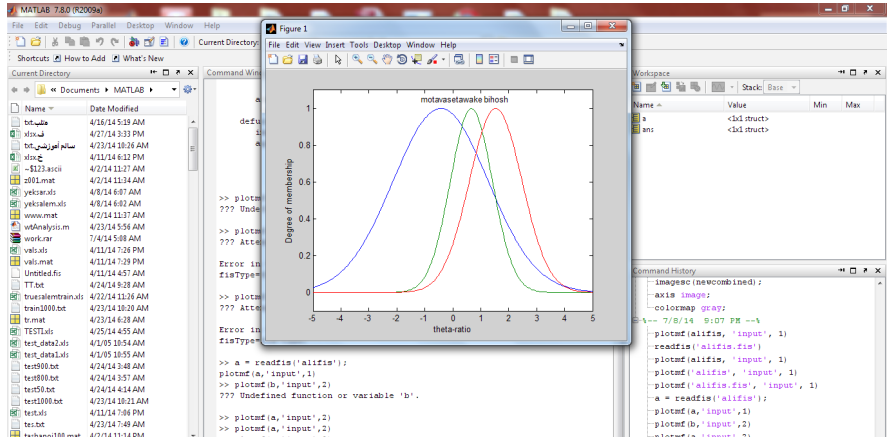

Fig. 1i. The third input in FIS

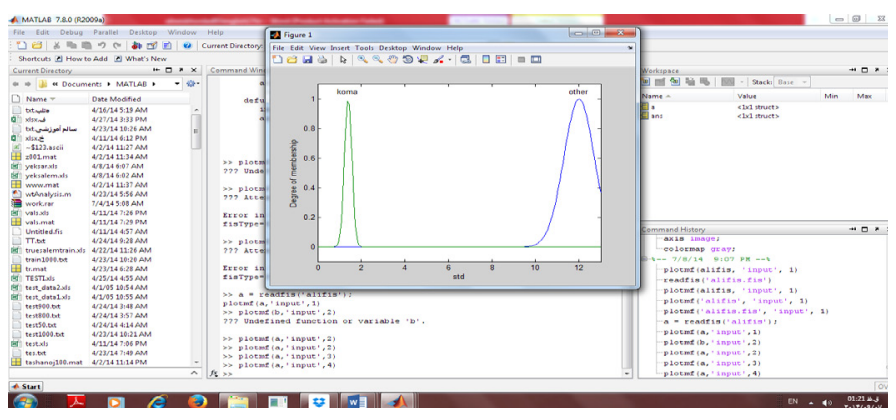

Fig. 1j. The fourth input in FIS.

In all, 24 fuzzy rules were composed regarding the four features of the input space. For the selected features and the defined target classes, an IF-THEN fuzzy rule was formulated, for each fuzzy sub-set, as follows $[14,15]$ :

$$
\text { if } x_{1} \text { is } A_{1}^{k} \text { and ... } x_{n} \text { is } A_{1}^{k} \text { then class } k
$$

The last phase of the study dealt with designing the ANFIS that is responsible for classifying the signals into four states of consciousness, moderate Anesthesia, deep Anesthesia, and coma. Compared to other fuzzy logic-based systems, one of the advantages of ANFIS is that no conversion of certain data into fuzzy data is required $[6,16]$. Moreover, parameters do not need to be initialized. In fact, first the fuzzy system is designed after which its membership functions are improved and tested using ANFIS. Finally, the trained network is used to distinguish different states of Anesthesia. All the membership functions drawn on in this system are Gaussian.

\section{RESULTS}

As stated in Section 2, the final ANFIS comprised four variables which had two, two, three, and two Gaussian membership functions, respectively. Accordingly, the total number of fuzzy rules amounted to 24 . In this study, $80 \%$ of the data collected was used for training and the remaining $20 \%$ was used for testing purposes. The assignment of data to train or test datasets was undertaken through simple randomization. An interesting finding in the present study was that the error rate of the model would decrease with an increase in the number of iterations.

The sensitivity rate of the proposed system was found to be $96.6 \%$, which simply means that it can recognize the Anesthesia states in $96.6 \%$ of the cases. Further, it was observed that the system could always (100\%) recognize Anesthesia accurately. Moreover, the accuracy rate of the proposed system was reported to be $98.45 \%$. 


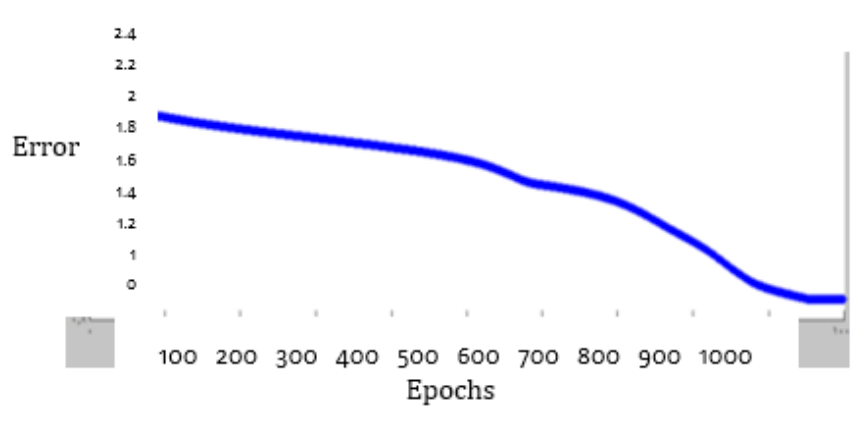

Fig. 2. The error rate of the system is negatively correlated with the number of iterations.

\section{CONCLUSION}

The system proposed in this study is an appropriate means of extracting useful EEG signals. Wavelet analysis was used here to describe EEG signals since it facilitates the decomposition of EEG signals into sub-bands. Further, the fact that each Anesthesia state has its own specific frequency bands enables the researcher to estimate the depth of Anesthesia in each state by finding the relationship between these frequency bands. To sum up, it appears that by combining the extracted statistical features from the wavelet coefficients of EEG signals and spectral analysis, it is possible to design a system which can accurately estimate the depth of Anesthesia. The proposed system can be of great help to anesthesiologists since it can recognize the Anesthesia states with high accuracy at a lower cost.

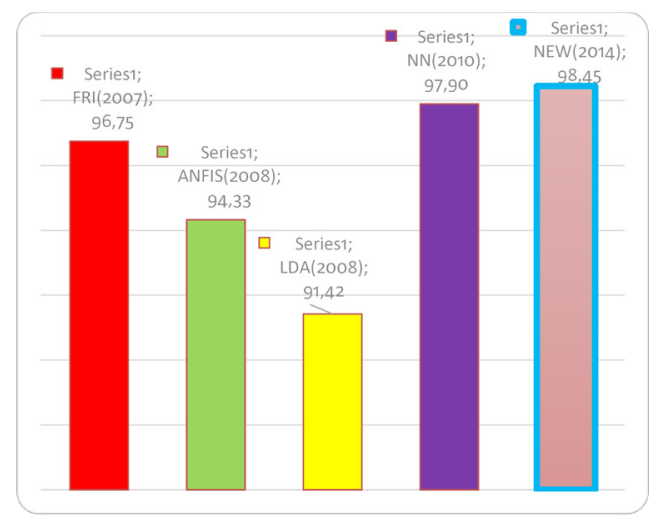

Fig. 3. Caparison between the proposed method and others.

The comparison of the statistical results from ANFIS to those from the previous studies - including neural networks, linear discernment, and even fuzzy models - reveals that the proposed fuzzy-neural system is advantageous for two reasons: First, it can estimate the depth of consciousness with high accuracy, and second, it has improved the deficiencies of the previous systems. This was facilitated through an optimized extraction of features using wavelet transform and formulating appropriate fuzzy rules. The low error rate of RMSE calculated for the system confirms the reliability of the results.

\section{REFERENCES}

[1] Krkic M, Roberts SJ, Rezek I, Jordan Ch. EEG-based assessment of anesthetic-depth using neural network. IEEE COLLOQUIUM ARTIF INTELL METHODS BIOSIGNAL ANAL 1996; 10: 1-6.

[2] Ye S, Choi S, Kim D. Analysis on the depth of anesthesia by using EEG and ECG signals. TRANS ELECTR ELECTRON MATER 2013; 6: 299303.

[3] ZoughiT,Boostani R. Presenting a combinatorial feature to estimate depth of anesthesia. INT J INFORM COMMUN ENG 2003; 2: 89-93.

[4] Kemal M, Kiymik A, Mehmet Akin B, Subasi A. Automatic recognition of alertness level by using wavelet transform and artificial neural network. J NEUROSCI METH 2004; 139: 231-240.

[5] Subasi A. Automatic recognition of alertness level from EEG by using neural network and wavelet coefficients. EXPERT SYST APPL2005; 28: 278-283.

[6] Goodarzi M, Freitas MP. Mia-qsar coupled to principal component analysis-adaptive neurofuzzy inference systems (pca-anfis) for the modeling of the anti-hiv reverse transcriptase activities of tibo derivatives. EUR J MED CHEM 2010; 2: 132-138.

[7] Buyukbingol E, Sisman A, Akyildiz M, Alparslan FN, Adejare A. Adaptive neuro-fuzz inference system (anfis): A new approach to predictive modeling in qsar applications: A study of neuro-fuzzy modeling of pcpbased nmda receptor antagonists. BIOORG MED CHEM LETT 2007; 12 : 4265-4282.

[8] Zhang XS, Roy RJ.Derived fuzzy knowledge model for estimating the depth of anesthesia.IEEE. TRANS BIOMED ENG 2001; 1: 312-323.

[9] QuianQuirogaa R, Sakowitzb OW, Basarce E, Schürmann M. Protocol wavelet transform in the analysis of the frequency composition of evoked potentials. BRAIN RES PROTOC 2001; 8: 16-24.

[10] Güler İ, Übeyli ED. Adaptive neuro-fuzzy inference system for classification of EEG signals using wavelet coefficients. J NEUROSCI METH 2005; 148: 113-121.

[11] Xizheng ZH, Ling Y, Weixiong W. Wavelet time-frequency analysis of electro-encephalogram (EEG) processing. INT J ADV COMP SCI APPL 2010; 5: 1-8.

[12] Esmaeili V, Assareh A, Shamsollahi MB, Moradi MH, Arefian NM. Designing a fuzzy rule based system to estimate depth of anesthesia. PROC IEEE S COMPUT INTELL DAT MIN2007; 5:681-687.

[13] Baig NM, Gholamhosseini H, Harrison MJ. Fuzzy logic based smart anesthesia monitoring system in the operation theatre. WSEAS TRANS CIRC SYST 2012; 11: 21-32.

[14] Nakashima T, Nakai G, Ishibuchi H. Improving the performance of fuzzy classification systems by membership function learning and feature selection. IEEE INT CONF FUZZY SYST, 2002; 1: 488-493.

[15] Ishibuchi H, Nozaki K, Yamamoto N, TanakaH. Selecting fuzzy if-then rules for classification problems using genetic algorithm. IEEE TRANS. FUZZY SYST 1995; 3: 260-270.

[16] Muthuswamy J, Roy RJ. The use of fuzzy integrals and bi-spectral analysis of the electroencephalogram to predict movement under anesthesia. IEEE TRANS BIOMED ENG 1999; 463: 291-299.

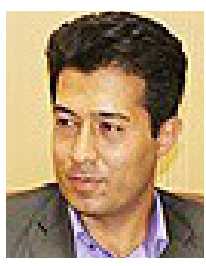

Mansour esmaeilpour, received the B.S. and M.S. degrees in Computer Engineering-Software Engineering in Iran, in 2002 and 2004, respectively. He also received the Ph.D. degree in Computer Engineering from The National University of Malaysia, in 2012. Currently, he is an assistant professor in Computer Engineering Department at Islamic Azad University, Hamedan Branch, Hamedan, Iran. As well as, he worked from 2013 to 2014 as Postdoctoral Researcher at National University of Malaysia. His research interests include Data mining, Process Mining, Learning Systems and Machine Learning.

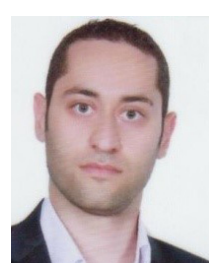

Ali Raeis Ali Mohammadi was born in 1986 in Mahallat, Iran. He received his B.Sc. in software engineering in 2007 from Khomein Branch, Islamic Azad University, Khomein, Iran and his M.Sc. in computer system design in 2013 from Arak Branch, Islamic Azad University, Arak, Iran. His research interests among others include medical signal processing and image processing. 